P719 PREVALENCE AND TYPE-SPECIFIC DISTRIBUTION OF ONCOGENIC HUMAN PAPILLOMAVIRUS AMONG FEMALE SEX WORKERS IN COTONOU, WEST AFRICA

${ }^{1}$ Fernand Guédou*, 'Luc Béhanzin, ${ }^{1} E l l a$ Goma-Matsétsé, 'Marlène Aza-Gnandji, ${ }^{1}$ Nassirou Geraldo, ${ }^{2}$ Fatoumata Korika Tounkara, ${ }^{3}$ Julie Guenoun, ${ }^{4}$ François Coutlée, ${ }^{5}$ Benjamin Hounkpatin, ${ }^{6}$ Michel Alary. ${ }^{1}$ Dispensaire IST, Cotonou, Benin; ${ }^{2}$ Axe Santé des Populations et Pratiques Optimales en Santé, HSS, Social and Preventive Medicine, Québec, Canada; ${ }^{3}$ Centre de Recherche du Centre Hospitalier de I'Université de Montréal, Département de Microbiologie et Infectiologie, Montréal, Canada; ${ }^{4}$ 'Université de Montréal, Microbiologie Médicale et Infectiologie, Montreal, Canada; ${ }^{5}$ University of Abomey Calavi, BP, Benin; ${ }^{6} \mathrm{CHU}$ de Quebec - Université Laval, Quebec, Canada

\subsection{6/sextrans-2019-sti.783}

Background Female sex workers (FSW) are at higher risk of Human Papillomavirus (HPV) infections. Yet, few data exist on the prevalence and the types of HPV circulating among them. Baseline data from FSW recruited in a longitudinal study were analyzed to determine the prevalence and type-specific distribution of oncogenic HPV among FSW in Cotonou.

Methods Data from 309 FSW with valid cervical specimens (out of 312 enrolled) were analyzed. Cervical specimens were processed through March 2019, using the Linear Array HPV genotyping test (LA-HPV) (Roche Molecular Systems). Where appropriate, a real-time PCR assay specific for type 52 was performed to control for cross-reactivity with HPV-33, 35 or 58. The overall and type-specific prevalence of oncogenic HPV were estimated according to the level of risk: high risk (HR-HPV) and low risk (LR-HPV).

Results The mean age of the 309 women at enrollment was $34.97( \pm 10.66)$ and that at their first intercourse was 17.53 $( \pm 2.66)$. Almost half of them $(45.8 \%)$ were Beninese and $25.8 \%$ were HIV positive. Condom use at the last sex with clients and boyfriend was reported by $97.7 \%$ and $14.5 \%$ of women, respectively. At least one HR-HPV was detected in 237 women $(88.3 \%)$ and the ten most frequent were HPV58 (37.5\%), HPV16 (36.6\%), HPV52 (28.8\%), HPV35 (23.3\%), HPV68 (22.0\%), HPV18 (20.7\%), HPV45 (15.2\%), HPV33 (11.0\%), HPV59 (9.1\%), HPV51 (6.5\%). LR-HPV were found in 186 women (60.2\%): HPV81 (23.6\%); HPV61 (23.0\%); HPV72 (15.2\%); VPH42 (12.0\%); VPH70 (8.4\%), VHP54 (5.8\%); VPH 6/VPH11 (5.5\%) and VPH40 (2.6\%). HR-HPV presence was not associated with HIV status $(p=0.897)$ while that of LR-HPV was $(p=0.037)$.

Conclusion To our knowledge, this study is the first to provide HPV data among FSW in West Africa. The high prevalence and atypical distribution of oncogenic HPV among this high risk population might have implications for vaccine design.

Disclosure No significant relationships.

\section{P722 AN UNUSUAL CONSTELLATION OF SYMPTOMS: OUTPATIENT DIAGNOSIS OF NEUROSYPHILIS WITH NO HISTORY OF PRIOR SYPHILIS SYMPTOMS}

Elizabeth Liu*, Eliza Newbury-Palma, Jacob Taylor, Anthony Izokaitis. Mercy Muskegon, Medical Education, Muskegon, USA

10.1136/sextrans-2019-sti.784

Background Neurosyphilis presents in early and later stages of syphilis. Cerebrospinal fluid, meninges and vascular structures are involved in the early stages of neurosyphilis, while in the late stage; cerebral tissue and spinal cord parenchyma are affected. Neurosyphilis can manifest with many different symptoms. Diagnosis criteria remains ambiguous and treatment options are classified very low quality of evidence by World Health Organization. We present a case of neurosyphilis with progressive sensory loss whose primary and secondary phases were not detected.

Methods 52 year old male initially presented with upper respiratory symptoms, headache, facial swelling, and visual disturbances. 20 pound weight loss, positive candida throat culture, diagnosis of panuveitis, and progressive hearing loss prompted diverse differential workup. Rapid plasma reagin and fluorescent treponemal antibody absorption were positive suggesting syphilis and with neurologic symptoms met neurosyphilis diagnosis. After penicillin treatment, symptoms resolved and labs showed disease resolution.

Results RPR titer high $1: 128$. In 2 months, WBC count increased from 9.8 to 22.0. CSF analysis showed a neutrophilic leukocytosis with elevated protein. Repeat CSF 6 months post-treatment showed negative VDRL CSF qualitative and titer negative. RPR 1:4. Syphilis antibody $\geq 70.0$ high.

Conclusion Neurosyphilis can be encountered in sensory, neuropsychiatric and neurologic domains. The incidence of syphilis in Michigan has increased from 365 to 480 cases and with a national 10.5\% increase from 2016 to 2017. In this case, neurosyphilis improvement was observed with resolution of sensory deficits with penicillin treatment. Impaired cognition or mood disturbance were not observed throughout the patient's clinical course. Attention should be given to progressive sensory loss because syphilis in its early stages can be overlooked, left untreated and can lead to irreversible manifestations. This case illustrates the need for further awareness on neurosyphilis as this disease can present in forms that are not common to what the current literature shows.

Disclosure No significant relationships.

\section{P724 A CONDITIONAL PAY-FOR-PERFORMANCE PROGRAM TO IMPROVE SYPHILIS SCREENING IN CHINESE STD CLINICS: A PILOT INTERVENTION}

${ }^{1}$ Shen Hongcheng, ${ }^{1}$ Cheng Wang, ${ }^{1}$ Shujie Huang, ${ }^{2}$ Christopher Weisen, ${ }^{1}$ Bin Yang, ${ }^{1} \mathrm{He}-$ Ping Zheng, ${ }^{3} \mathrm{M}$ Kumi Smith*. 'Guangdong Center for STD Control and Prevention, Guangzhou, China; ${ }^{2}$ University of North Carolina Chapel Hill, Chapel Hill, USA; ${ }^{3}$ University of Minnesota Twin Cities, Epidemiology and Community Health, Minneapolis, USA

\subsection{6/sextrans-2019-sti.785}

Background Under-screening of syphilis in clinical settings is a pervasive problem, especially in resource constrained settings where heavy patient loads and competing health priorities inhibit health providers' ability to meet screening coverage targets. We piloted a "conditional pay-for-performance (P4P) strategy," which rewarded providers at sexually transmitted disease (STD) clinics a monetary bonus for every confirmed case of syphilis they screened. By tying rewards to the number of cases detected, this strategy seeks to incentivize providers to concentrate their counseling efforts on indicated patients who need it the most.

Methods Five STD clinics in a high syphilis transmission setting of China participated in the 6-month intervention. Data 
from the pre-intervention period was abstracted from clinic records. Multilevel logistic regression models with random intercepts to account for clustering within clinics were used to compare rates of syphilis case detection (number of confirmed positive cases over the total number of clinic attendees) in the 6-month periods prior to and during the intervention. Estimates were adjusted for age and sex of clinic attendees.

Results A total of 7900 patients (49.0\% male; 17.7\% under the age of 25) sought care at one of the five STD clinics over the course of the study. Adjusted odds of a positive syphilis screen were greater during the intervention period compared to the pre-intervention interval (odds ratio, 1.33; $95 \%$ confidence interval, 1.14-1.56). Variability in clinic-level effects were substantial given the small number of sites of this pilot study.

Conclusion Results of a conditional pay-for-performance pilot study demonstrate the feasibility and preliminary effectiveness of a conditional P4P strategy to improve syphilis case detection in Chinese clinical settings. Plans are underway for a fully powered randomized trial, findings from which could inform the utility of this approach for improving detection of common STDs in other resource constrained settings.

Disclosure No significant relationships.

\section{P725 FREQUENCY AND CHARACTERISTICS OF BIOLOGIC FALSE POSITIVE TESTS FOR SYPHILIS, REPORTED IN FLORIDA AND NEW YORK CITY, 2013-2017}

\footnotetext{
${ }^{1}$ James Matthias*, ${ }^{2}$ Ellen Klingler, ${ }^{3} J u l i a$ Schillinger, ${ }^{4}$ Thomas Peterman, ${ }^{5}$ Craig Wilson. ${ }^{1}$ Centers for Disease Control and Prevention, Division of STD Prevention, Tallahassee, USA; ${ }^{2}$ New York City Department of Health and Mental Hygiene, New York City, USA; ${ }^{3}$ Centers for Disease Control and Prevention, New York City, USA; ${ }^{4}$ Centers for Disease Control and Prevention, Division of STD Prevention, Atlanta, USA; ${ }^{5}$ Florida Department of Health, STD and Viral Hepatitis Section, Tallahassee, USA

\subsection{6/sextrans-2019-sti.786}

Background Biologic false positive (BFP) non-treponemal test results, defined as specimens with reactive non-treponemal (NT) and non-reactive treponemal test results are received and processed by United States public health surveillance programs. Non-treponemal BFPs can be attributed to a variety of infectious and non-infectious diseases. However, little is known about the distribution of BFP NT titers. We describe the frequency, NT titer distribution, and descriptive characteristics of people with BFP NT results reported in Florida and New York City (NYC).

Methods Reactive NTs and BFPs (reactive NT test and $\geq 1$ non-reactive treponemal tests from a person with no past or current reactive treponemal test) were extracted from Florida's and NYC's sexually transmitted diseases surveillance systems for 2013-2017. Results were de-duplicated by specimen collection date, test type, and titer value. For individuals, BFPs were stratified by site, patient characteristics, and highest reported titer during study period.

Results A total of 526,540 reactive NTs were reported. Among these were 57,850 BFPs (28,183 Florida and 29,397 NYC), $11 \%$ of all reactive NTs, from 39,920 individuals $(19,313$ Florida and 20,607 NYC). Titers of $1: 1$ accounted for $55 \%$ $(n=31,580)$ of all BFPs, but 5,250 (9\%) were $\geq 1: 8$, including $654(1.1 \%) \geq 1: 32$. Persons with BFP were most often women $68 \%(n=27,161 / 39,920)$. Individuals $40+$ years were at increased odds (OR 2.12; 95\% C.I. 1.78-2.55) of having a high titer $\mathrm{BFP}(\geq 1: 32$ titer).

Conclusion Syphilis BFPs (non-cases) account for a substantial number of reported tests that require processing. Some countries classify titers $\geq 1: 8$ as presumptive syphilis without treponemal testing, thus may over-count cases. Areas requiring both treponemal and NT tests for syphilis case reporting may benefit from requiring laboratories to report negative treponemal tests when they are associated with reactive NT tests. Review of patient histories might identify underlying conditions that contribute to high-titer BFP results.

Disclosure No significant relationships.

\section{P726 SYPHILIS - DO WE SEE THE END OF THE STEEP RISE IN CASES IN GERMANY?}

${ }^{1}$ Klaus Jansen*, ${ }^{2}$ Viviane Bremer. ${ }^{1}$ Robert Koch Institute, Infectious Disease Epidemiology, Berlin, Germany; ${ }^{2}$ Robert Koch Institute, Berlin, Germany

\subsection{6/sextrans-2019-sti.787}

Background The number of reported syphilis cases in Germany doubled between 2001-2004 to over 3,000/year and remained stable until 2009. Between 2010 and 2016, the annual increase was between $7 \%$ and $22 \%$. We analysed syphilis surveillance data to assess characteristics of current epidemiological dynamics in order to initiate appropriate prevention measures.

Methods Since 2001, laboratories are required to notify syphilis diagnoses anonymously, physicians complement clinical information. Potential double notifications are identified. Since 2016, HIV-status is reported. We analysed syphilis cases by year of diagnosis, age, sex, area of residence, and transmission category.

Results 7,520 cases were reported in 2017, corresponding to a $4.5 \%$ rise compared to 2016. Incidence was 9.1/100,000 inhabitants, with highest incidences in metropolitan cities as Cologne (39.4), Berlin (37.9), and Munich (35.0). From January to June 2018, syphilis cases dropped by $4.3 \%$ compared to this period in 2017. Men accounted for $94 \%$ of cases in 2017. $83 \%$ of cases with information on transmission route were men who have sex with men (MSM), 17\% heterosexuals. The proportion of MSM aged 40 years or above was continuously above $45 \%$ since 2007 . Syphilis reinfection was reported for 27\% HIV-negative MSM and 63\% HIV-positive MSM. HIV-coinfection was reported for 48\% MSM and 7\% heterosexuals. HCV-coinfection was reported for 7\% HIV-positive MSM and $1 \%$ of HIV-negative MSM.

Conclusion Data showed a high burden of disease in MSM in metropolitan cities and higher age groups. Increasing risk behavior due to HIV pre-exposure prophylaxis (PrEP) could impact the syphilis epidemic in Germany in specific groups, considering the high proportion of Syphilis reinfections as well as a distinct rate of HCV-coinfection. To avoid negative effects of PrEP for the Syphilis epidemic, targeted and innovative approaches to foster early screening and treatment, like internet counselling, home sampling, home testing and broadening venue-based (rapid) testing should be discussed.

Disclosure No significant relationships. 